Original paper

\title{
FLUKA simulation of target fragmentation in proton therapy
}

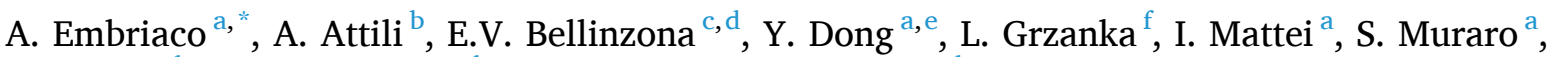 \\ E. Scifoni ${ }^{\mathrm{d}}$, F. Tommasino ${ }^{\text {c,d }}$, S.M. Valle ${ }^{\mathrm{a}}$, G. Battistoni $^{\mathrm{a}, \mathrm{d}}$ \\ ${ }^{a}$ Istituto Nazionale di Fisica Nucleare, Sezione di Milano, Italy \\ ${ }^{\mathrm{b}}$ Istituto Nazionale di Fisica Nucleare, Sezione di Roma Tre, Italy \\ ${ }^{\mathrm{c}}$ Universitá degli studi di Trento, Italy \\ d TIFPA Trento Institute for Fundamental Physics and Application, Italy \\ ${ }^{\mathrm{e}}$ Universitá degli studi di Milano, Italy \\ ${ }^{\mathrm{f}}$ Institute of Nuclear Physics, Kraków, Poland
}

\section{A R T I C L E I N F O}

\section{Keywords:}

Proton therapy

Target fragmentation

Monte Carlo simulation

\begin{abstract}
A B S T R A C T
In proton therapy, secondary fragments are created in nuclear interactions of the beam with the target nuclei. The secondary fragments have low kinetic energies and high atomic numbers as compared to primary protons. Fragments have a high LET and deposit all their energy close to the generation point. For their characteristics, secondary fragments can alter the dose distribution and lead to an increase of RBE for the same delivered physical dose. Moreover, the radiobiological impact of target fragmentation is significant mostly in the region before the Bragg peak, where generally healthy tissues are present, and immediately after Bragg peak. Considering the high biological impact of those particles, especially in the case of healthy tissues or organs at risk, the inclusion of target fragmentation processes in the dose calculation of a treatment planning system can be relevant to improve the treatment accuracy and for this reason it is one of the major tasks of the MoVe IT project.

In this study, Monte Carlo simulations were employed to fully characterize the mixed radiation field generated by target fragmentation in proton therapy. The dose averaged LET has been evaluated in case of a Spread Out Bragg Peak (SOBP). Starting from LET distribution, RBE has been evaluated with two different phenomenological models. In order to characterize the mixed radiation field, the production cross section has been evaluated by means of the FLUKA code. The future development of present work is to generate a MC database of fragments fluence to be included in TPS.
\end{abstract}

\section{Introduction}

In the interaction with the biological tissues, protons lose energy mainly by means of electromagnetic Coulomb interactions with electrons. The rate of energy loss per unit mass increases with depth as particles slow down reaching a maximum known as Bragg peak. In addition, nuclear interactions can take place with the atomic nuclei of the target material. At therapeutic energies of proton beam (60-250 $\mathrm{MeV}$ ), only target fragmentation can occurs.

Target fragments created in inelastic interactions of the proton beam with the target nuclei have low kinetic energy, high atomic number and high LET [1] as compared to primary protons [2]. So that, fragments alter the dose distribution, due to their short ranges that can spread in the order of $10-100 \mu \mathrm{m}[3,4]$.

Target fragmentation increases RBE along the treatment irradiation field with LET, as shown in several radiobiological measurements [5]. These characteristics make target fragmentation biologically relevant mostly in the region before the Bragg peak, where the cell inactivation due to ionization processes are less than nuclear interaction as compared to the Bragg peak region [3]. It results in RBE values significantly different from 1.1. i.e. the constant value currently used in proton therapy treatments, and does not consider target fragmentation. The topic of RBE variability in proton therapy is being widely debated in recent years, within this field, a widely supported hypothesis [3] is that secondary particles produced in target fragmentation could be one of the causes contributing to the increase of proton RBE [6].

The production cross sections of the target fragments are a topic of great interest but poor data are available: the direct measurement of target fragments is extremely difficult due to the short range of produced secondaries that have low probability to escape the target. The FOOT

\footnotetext{
* Corresponding author.

E-mail address: alessia.embriaco@mi.infn.it (A. Embriaco).
} 
(FragmentatiOn Of Target) experiment [7-10] plans to use a complex experimental setup and an inverse kinematic approach to investigate the target fragments.

In the MoVe IT project [11], the effect of target fragmentation will be included in TRiP98 $[12,13]$ TPS; the code is able to take into account the mixed radiation field for the description of biological effects of target fragmentation. In order to implement the transport of fragments in the TPS, a database for fragments fluence will be created.

In this paper, Monte Carlo (MC) simulations are performed to evaluate the amount of those target fragments with respect to primary protons fluence. The dose averaged LET has been evaluated in case of Spread Out Bragg Peak (SOBP) as well as the relative biological effectiveness by using the phenomenological models of Wedenberg [14] and McNamara [15]. The production cross sections of fragments at therapeutic energies have been evaluated by means of the FLUKA MC code. As the further step, FLUKA fluence spectra will be imported in TRiP98 TPS to evaluate the biological impact of fragmentation with a mixed field theory approach [13].

\section{MoVe IT project}

The aim of MoVe IT (Modeling and Verification for Ion beam Treatment planning) project is developing and testing innovative treatment planning models for particle therapy, accounting for a higher complexity of biophysical processes. The main effects that are going to be explored and implemented in MoVe-IT are: the biological impact of target nuclei fragmentation, RBE and intra-tumor heterogeneity.

In MoVe-IT project, the TPS modelling part is based on the use of TRiP98 (Treatment Planning for Particles) [12,13]: the first TPS for actively scanned heavy ions developed in the GSI pilot project on carbon ion therapy. Depth dose distribution, nuclear fragments spectra and stopping power data are the input of TPS. The biological effects of target implemented in FLUKA. For the water phantom, we have defined the mean ionization potential as $77 \mathrm{eV}$ using the MAT-PROP card to be consistent with TRiP98 value [19]. The FLUKA simulations were performed with $10^{9}$ simulated particles, in 10 batches of $10^{8}$ histories each.

\section{Dose averaged LET and RBE evaluation}

The dose averaged linear energy transfer $\left(L E T_{D}\right)$ is frequently used as a physical quantity to describe the biological effectiveness of mixed radiation fields [20]. $L E T_{D}$ is the dose-weighted mean value of the particle LET distribution at depth $z$ in the radiation field consisting of dose contribution $D_{i}$ from all particle species $i$ :

$\operatorname{LET}_{D}(z)=\frac{\Sigma_{i} \int L E T_{i}(E) D_{i}(E, z) d E}{\Sigma_{i} \int D_{i}(E, z) d E}=\frac{\Sigma_{i} \int L E T_{i}^{2}(E) \phi_{i}(E, z) d E}{\Sigma_{i} \int L E T_{i}(E) \phi_{i}(E, z) d E}$

The quantities $\operatorname{LET}_{i}(E)$ and $\phi_{i}(E, z)$ can be evaluated by FLUKA code and $L E T_{D}$ can been calculated using Eq. 1.

A Spread Out Bragg peak was simulated to homogeneously cover a $5 \times 5 \times 5 \mathrm{~cm}^{3}$ target of water placed at $10<z<15 \mathrm{~cm}$. The initial proton energies were equally spaced in the range of $115.52<E<145.49$ $\mathrm{MeV}$.

The evaluated dose distribution and the dose averaged LET as a function of depth are reported in Fig. 1. As expected, the dose averaged LET increases swiftly at the depth where the dose drops.

Literature offers several models to predict proton RBE based on dose averaged LET, dose and the tissue specific parameters $\alpha / \beta$ of the linearquadratic model [21]. The most used phenomenological RBE models are Wedenberg [14], Wilkens [22], McNamara [15] and Carabe [23].

Starting from the dose averaged LET obtained with FLUKA simulation, the RBE has been calculated considering Wedenberg and McNamara models.

In the Wedenberg model, RBE is given by the formula [14]:

$\operatorname{RBE}\left(\operatorname{LET}_{D}, D,(\alpha / \beta)_{p h}\right)=-\frac{1}{2 D}\left(\frac{\alpha}{\beta}\right)_{p h}+\frac{1}{D} \sqrt{\frac{1}{4}\left(\frac{\alpha}{\beta}\right)_{p h}^{2}+\left(q L E T_{D}+\left(\frac{\alpha}{\beta}\right)_{p h}\right) D+D^{2}}$

fragmentation can be evaluated as for projectile fragments using the available RBE model, in particular including LEMIV. Considering the contribution of each single fragment produced by a proton in water, the evaluation of total RBE is based on a mixed field approach [13].

\section{FLUKA simulation}

A Monte Carlo study has been performed with the FLUKA [16,17] where $q$ is a parameter obtained by experimental data, different for each cell line (for V79 cell line $q=0.434$ ) and $\alpha / \beta$ represents the intrinsic radio-sensitivity of the irradiated cells [24].

Recently the RBE model of McNamara [15] has been derived considering the most comprehensive collection of proton RBE experimental data:

$$
R B E\left(L E T_{D}, D,(\alpha / \beta)_{p h}\right)=\frac{1}{2 D}\left(\sqrt{\left(\frac{\alpha}{\beta}\right)_{p h}^{2}+4 D\left(\frac{\alpha}{\beta}\right)_{p h}\left(p_{0}+\frac{p_{1}}{(\alpha / \beta)_{p h}} L E T_{D}\right)+4 D^{2}\left(p_{2}+p_{3} \sqrt{\left(\frac{\alpha}{\beta}\right)_{p h}} L E T_{D}\right)^{2}}-\left(\frac{\alpha}{\beta}\right)_{p h}\right)
$$

code which is often used as a reference in hadrontherapy [18] and has been benchmarked against measured data.

All simulations were performed with developer version using the PRECISION defaults card, physics card COALESCE to activate the coalescence mechanism and EVAPORATE card with evaporation of heavy fragments, which is recommended for the study of fragments production in proton therapy. The materials used are the default materials where $p 1, p 2, p 3$ are experimental derived parameters assuming, for the pooled ensemble of cell lines, values of $p_{0}=0.99064, p_{1}=0.35605$, $p_{2}=1.1012$ and $p_{3}=0.0038703$.

In this work, the RBE has been calculated for the prescribed dose of interest of $2 \mathrm{~Gy}$ for a standard reference cell line, i.e. V79 (Chinese Hamster) $\alpha / \beta=2.7$ Gy [14].

In Fig. 2, the RBE has been evaluated with two different models and 


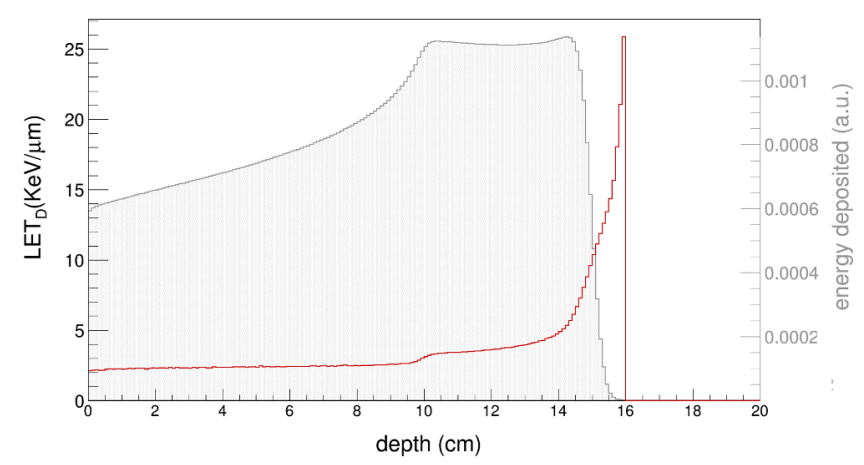

Fig. 1. Energy deposited in target volume as function of depth (grey) and the dose averaged LET (red) in case of SOBP (the initial beams energy is in the range of $115.52<E<145.49 \mathrm{MeV}$ ).

both have been calculated by considering the full radiation field (red lines) and only the primary protons (blue lines). In all analysed case, the RBE evaluated using Wedenberg is lower than McNamara in the region before the Bragg peak, while predicts higher RBE values in the distal region where both models significantly exceed the constant value.

The effect of the fragments considerations instead of only primary field is highlighted in the region before the Bragg peak and in the distal edge of SOBP (at depth $15<z<16 \mathrm{~cm}$ ), while becomes less evident in within SOBP where the predominant effect is the primary proton dose deposition. These results underline an underestimation of the biological dose in the entrance region and in the distal edge of SOBP, that as a potential consequence, in the healthy tissues may impact Normal Tissue Complication Probability (NTCP).

Therefore, an accurate knowledge of the target fragments production cross section is a fundamental requirement to correctly assess the biological dose of a proton beam. Waiting for FOOT experimental data [7], the different particle fluences of the mixed field has been studied for this purpose with FLUKA.

\section{Production cross section}

In order to estimate the contribution of target fragmentation, the production cross section due to inelastic interaction induced by proton beam in water has been evaluated using FLUKA code. The simulation is performed with proton pencil beams of different initial kinetic energies impinging on a thin water target. The target is a sphere of water with radius of $100 \mu \mathrm{m}$. The considered initial kinetic energies are 50, 100, 150,200 and $250 \mathrm{MeV}$ in order to cover the range of therapeutic energies. The production cross section of each fragment produced by inelastic interaction in water has been scored. FLUKA is able to score the energy distribution by particle type with the USRYIELD card combined

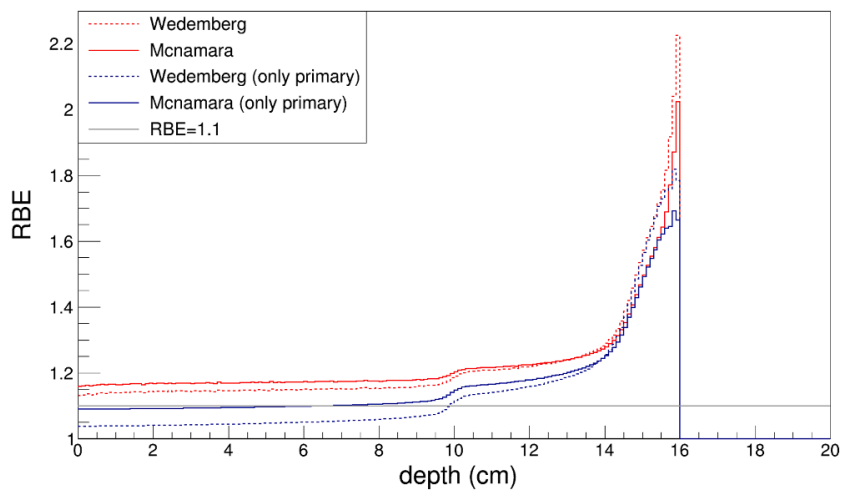

Fig. 2. RBE as function of depth evaluated with Wedenberg (dashed line) and McNamara Models (solid line) considering the full radiation field (red) and the primary protons (blue). The grey line represent the constant $\mathrm{RBE}=1.1$ adopted in clinical treatments.
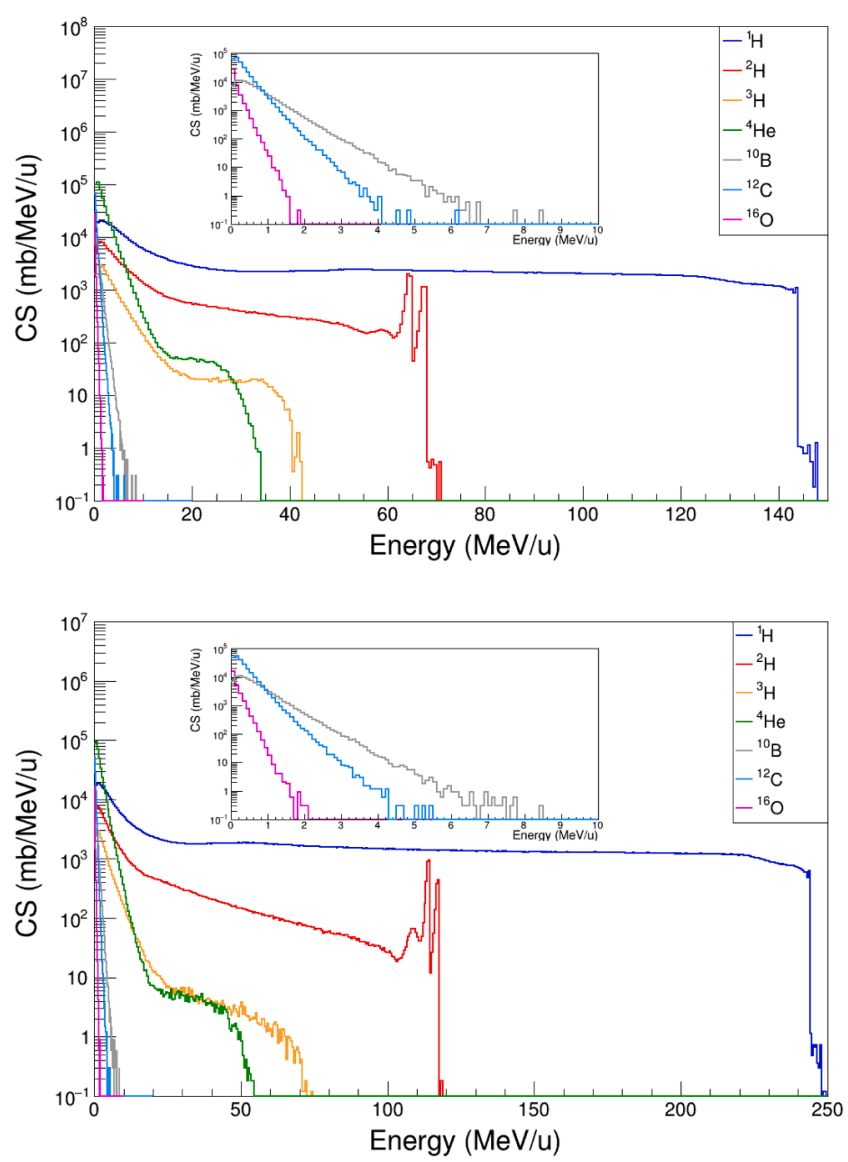

Fig. 3. Production cross section (CS) of inelastic interaction induced by a proton beam of $150 \mathrm{MeV}$ (top) and $250 \mathrm{MeV}$ (bottom) in water. The plots are in logarithmic scale.

with an AUXSCORE card. The AUXSCORE allow to select and distinguish the contribution of different particles.

In Fig. 3, the energy distribution of main fragments produced by proton beam of 150 and $250 \mathrm{MeV}$ has been reported. In Table 1, the mean energies of each fragment are reported.

From these results, it emerges that fragments of ${ }^{4} \mathrm{He},{ }^{10} \mathrm{~B},{ }^{12} \mathrm{C}$ and ${ }^{16} \mathrm{O}$ have low kinetic energies (few $\mathrm{MeV}$ ) as reported in $[3,4]$. The production cross section evaluated with FLUKA will be validated with forthcoming experimental data acquired by FOOT and can be imported in the TPS for the transport of target fragments.

\section{Fragments fluence}

In order to include the impact of fragmentation and estimate the biological contribution of fragments in TPS, the secondary particles production has been evaluated with FLUKA code. In the simulation, the

\section{Table 1}

Mean energy $\left(E_{M}\right)$ of main fragments produced by proton beam of 150 and 250 $\mathrm{MeV}\left(E_{p}\right)$ in water evaluated with FLUKA MC code.

\begin{tabular}{ccc}
\hline Fragment & $\begin{array}{c}E_{p}=150 \mathrm{MeV} \\
E_{M}(\mathrm{MeV} / \mathrm{u})\end{array}$ & $\begin{array}{c}E_{p}=250 \mathrm{MeV} \\
E_{M}(\mathrm{MeV} / \mathrm{u})\end{array}$ \\
\hline${ }^{1} \mathrm{H}$ & 48.5 & 82.8 \\
${ }^{2} \mathrm{H}$ & 15.2 & 17.2 \\
${ }^{3} \mathrm{H}$ & 3.9 & 3.8 \\
${ }^{4} \mathrm{He}$ & 1.7 & 1.7 \\
${ }^{10} \mathrm{~B}$ & 0.6 & 0.6 \\
${ }^{12} \mathrm{C}$ & 0.3 & 0.3 \\
${ }^{16} \mathrm{O}$ & 0.1 & 0.1 \\
\hline
\end{tabular}




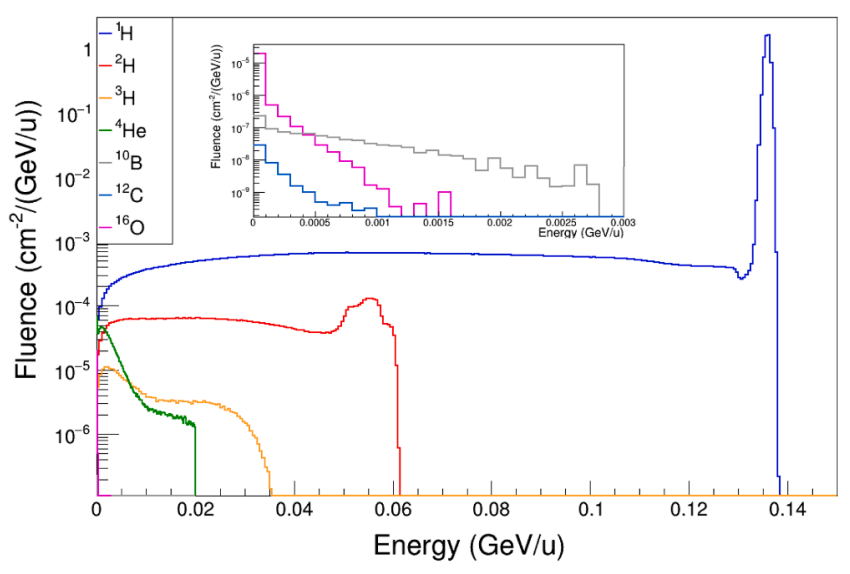

(a)

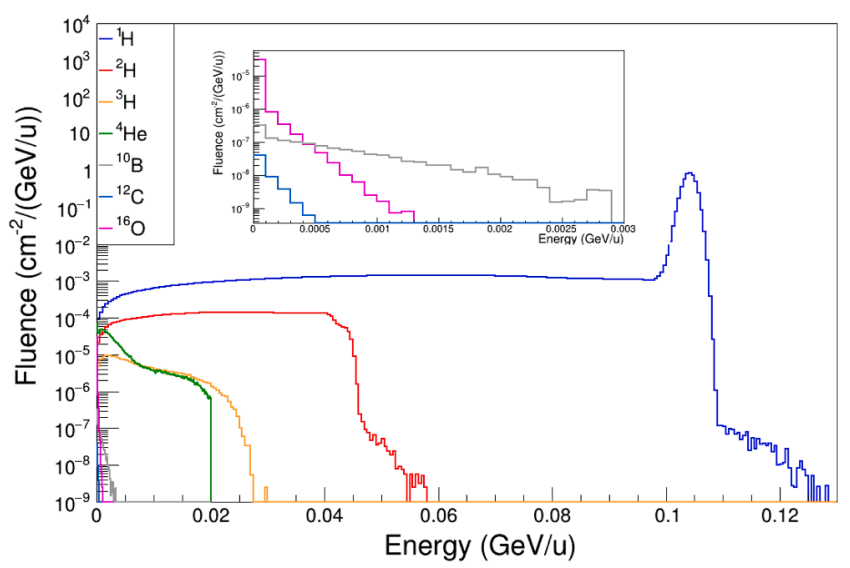

(b)

Fig. 4. Fluence of target fragments induced by $150 \mathrm{MeV}$ protons in water at $\mathrm{z}$ $=2.5$ (a) and $7.5 \mathrm{~cm}$ (b) (Bragg Peak at $15.8 \mathrm{~cm}$ ).

source is a monoenergetic proton beam of $150 \mathrm{MeV}$. The water phantom is a cylinder of radius $20 \mathrm{~cm}$. The scored quantity is the fluence of each fragments at different depths in a water phantom. FLUKA scores the track-length fluence distribution of certain particle with the USRTRACK card combined with an AUXSCORE card. The fluence has been evaluated at different depths in a volume (radius $\mathrm{r}=20 \mathrm{~cm}$, height $\mathrm{z}=0.1 \mathrm{~cm}$ and volume $\mathrm{V}=125.6 \mathrm{~cm}^{3}$ ): the radius of target was chosen in a way that the full energy of the beam would be deposited inside the volume.

In Figs. 4 and 5, the fluence of fragments produced by a proton beam of $150 \mathrm{MeV}$ in water has been reported at different depths. The fluence of all protons is characterized by two components: the primary particles (the region with peak) and the secondaries protons (the plateau region). Due to the energy dependence of the nuclear interaction, the energies of fragments are higher in the entrance channel than the Bragg peak region and decrease with increasing depth. In Bragg region, the effect of fragmentation is less important than ionization processes and the produced fragments have such a low energy to become inefficient biologically [3]. Therefore, target fragmentation is more relevant in the region before the Bragg peak.

From the results shown in Figs. 4 and 5, it emerges that the main contributors to the target fragmentation are Hydrogen isotopes, according to $[25,26]$, but a significant contribution to the dose distribution is given also by Helium fragments, as shown in [27].

\section{Conclusions}

The full particle spectrum of proton beams at therapeutic energies in water has been presented and characterized in this work based on a

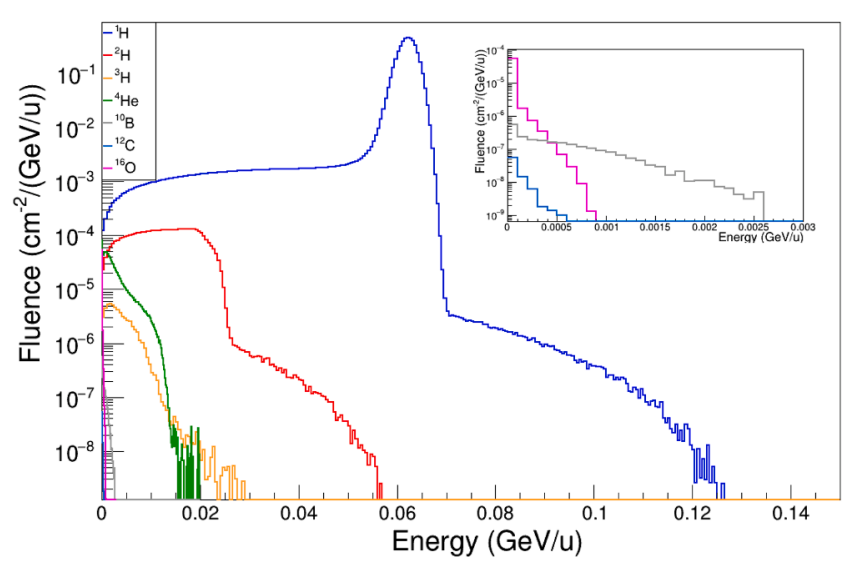

(a)

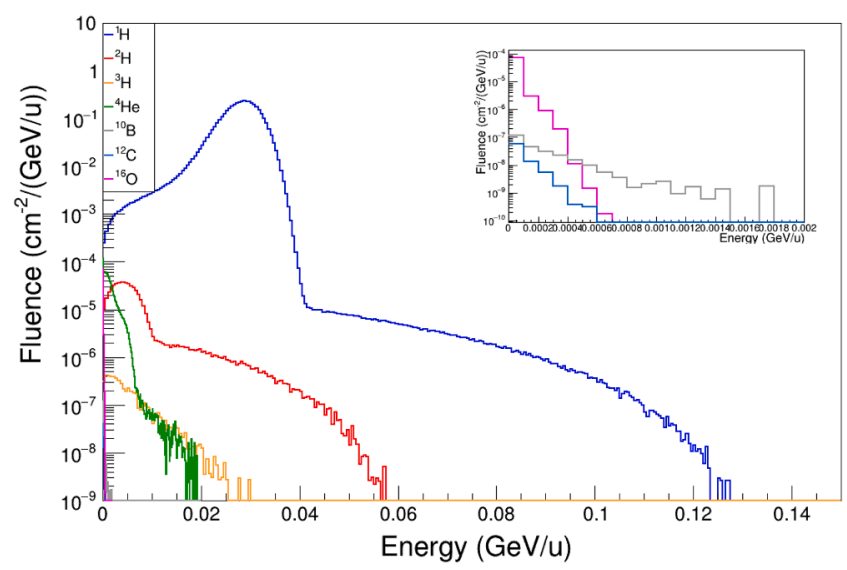

(b)

Fig. 5. Fluence of target fragments induced by $150 \mathrm{MeV}$ protons in water at 12.5 (a) and $15 \mathrm{~cm}$ (b) (Bragg Peak at $15.8 \mathrm{~cm}$ ).

FLUKA MC study. For most abundant target fragments, fluence has been studied as well as cross sections and mean production energy, to allow evaluation of the relevance of these secondary particles in a biological dose calculation. Simulations results evidence that secondary protons are the most abundant target fragments; their production rate is two orders of magnitudes higher than ${ }^{4} \mathrm{He}$, the second most relevant species.

A first estimation of the biological impact is given by evaluating the dose averaged LET for a SOBP in therapeutic energy range, with Wedenberg [14] and McNamara [15] RBE models. RBE results to be grater than 1.1 in the entrance channel, before reaching the well known maximum in the distal edge of SOBP, underlining an underestimation of the biological dose in the healthy tissue region.

For a more dedicated analysis of the biological impact a full mixed field approach is necessary, beyond the pure $L E T_{D}$ based approximation, which has been reported to be often insufficient [28]. In the framework of the MoVe IT project, on the basis of these simulations, the creation of a detailed fragments database is now possible for the inclusion of target fragmentation effect in a a biological TPS. Using TRiP98, the biological effects of target fragmentation can be evaluated with LEMIV model. A systematic analysis of the biological impact of such fragments, with a mixed field approach, will be subject of a forthcoming paper.

\section{Declaration of Competing Interest}

The authors declare that they have no known competing financial interests or personal relationships that could have appeared to influence the work reported in this paper. 


\section{Acknowledgment}

This work was supported by Istituto Nazionale di Fisica Nucleare (project MoVe-IT).

\section{References}

[1] Kodaira S, Yasuda N, Konishi T, Kitamura H, Kurano M, Kawashima H, Uchihori Y, Ogura K, Benton E. Calibration of CR-39 with atomic force microscope for the measurement of short range tracks from proton-induced target fragmentation reactions. Rad Meas 2013;50:232-6.

[2] Cucinotta FA, Katz R, Wilson JW, Townsend LW, Shinn J, Hajnal F. Biological effectiveness of high-energy protons: target fragmentation. Rad Res 1991;127(2): 130-7.

[3] Tommasino F, Durante M. Proton Radiobiology. Cancers 2015;7(1):353-81. https://doi.org/10.3390/cancers7010353. ISSN 2072-6694.

[4] Embriaco A, Dong Y, Mattei I, Muraro S, Valle S, Battistoni G. Target fragmentation in protontherapy. IL NUOVO CIMENTO 2018;100(207):41.

[5] Grün R, Friedrich T, Krämer M, Scholz M. Systematics of relative biological effectiveness measurements for proton radiation along the spread out Bragg peak: experimental validation of the local effect model. Phys Med Biol 2017;62(3):890.

[6] Paganetti H. Nuclear interactions in proton therapy: dose and relative biological effect distributions originating from primary and secondary particles. Phys Med Biol 2002;47(5):747.

[7] Alexandrov A, Alunni Solestizi L, Ambrosi G, Argirò S, Arteche Diaz R, Bartosik N, Battistoni G, Belcari N, Bellinzona E, Bianucci S, et al. The foot experiment: fragmentation measurements in particle therapy.

[8] Montesi M, Lauria A, Alexandrov A, Alunni Solestizi L, Giovanni A, Argirò S, Diaz R, Bartosik N, Battistoni G, Belcari N, Bellinzona E, Bianucci S, Biondi S, Giuseppina M, Bruni G, Camarlinghi N, Carra P, Cerello P, Ciarrocchi E, Lellis G. Ion charge separation with new generation of nuclear emulsion films, Open. Physics 2019;17:233-40. https://doi.org/10.1515/phys-2019-0024.

[9] Morrocchi M, Ciarrocchi E, Alexandrov A, Alpat B, Ambrosi G, Argirò S, Diaz RA Bartosik N, Battistoni G, Belcari N, et al. Development and characterization of a $\triangle \mathrm{E}-\mathrm{TOF}$ detector prototype for the FOOT experiment. Nucl Instrum Methods Phys Res Sect A 2019;916:116-24.

[10] Valle SM, Biondi S, Mattei I, Bisogni M, Pullia M, Camarlinghi N, Sitta M, Hild S, Garbini M, Francesconi M, et al. The FOOT (FragmentatiOn Of Target) experiment. Nuovo Cim 2019;41:169.

[11] MoVe IT project.https://www.tifpa.infn.it/projects/move-it/, 2020.

[12] Krämer M, Scholz M. Treatment planning for heavy-ion radiotherapy: calculation and optimization of biologically effective dose. PMB 2000;45(11):3319-30. https://doi.org/10.1088/0031-9155/45/11/314.
[13] Krämer M, Scholz M. Rapid calculation of biological effects in ion radiotherapy. Phys Med Biol 2006;51(8):1959-70. https://doi.org/10.1088/0031-9155/51/8/ 001.

[14] Wedenberg M, Lind BK, Hårdemark B. A model for the relative biological effectiveness of protons: the tissue specific parameter $\alpha / \beta$ of photons is a predictor for the sensitivity to LET changes. Acta Oncol 2013;52(3):580-8.

[15] McNamara AL, Schuemann J, Paganetti H. A phenomenological relative biological effectiveness (RBE) model for proton therapy based on all published in vitro cell survival data. Phys Med Biol 2015;60(21):8399.

[16] Battistoni G, Mattei I, Muraro S. Nuclear physics and particle therapy. Adv Phys: X 2016;1(4):661-86. https://doi.org/10.1080/23746149.2016.1237310.

[17] Ferrari A, Sala P, Fasso A, Ranft J. FLUKA: A multi-particle transport code (Program version 2005) [Tech. Rep.]; 2005.

[18] Böhlen T, Cerutti F, Chin M, Fassò A, Ferrari A, Ortega P, Mairani A, Sala PR, Smirnov G, Vlachoudis V. The FLUKA code: developments and challenges for high energy and medical applications. Nucl Data Sheets 2014;120:211-4.

[19] Krämer M, Jäkel O, Haberer T, Kraft G, Schardt D, Weber U. Treatment planning for heavy-ion radiotherapy: physical beam model and dose optimization. Phys Med Biol 2000;45(11):3299.

[20] Grassberger C, Trofimov A, Lomax A, Paganetti H. Variations in linear energy transfer within clinical proton therapy fields and the potential for biological treatment planning. Int J Rad Oncol Biol Phys 2011;80(5):1559-66.

[21] Carante MP, Ballarini F. Modelling cell death for cancer hadrontherapy. AIMS Biophys 2017;4:465-90.

[22] Wilkens JJ, Oelfke U. Analytical linear energy transfer calculations for proton therapy. Med Phys 2003;30(5):806-15.

[23] Carabe A, Moteabbed M, Depauw N, Schuemann J, Paganetti H. Range uncertainty in proton therapy due to variable biological effectiveness. Phys Med Biol 2012;57 (5):1159.

[24] Van Leeuwen C, Oei A, Crezee J, Bel A, Franken N, Stalpers L, Kok H. The alfa and beta of tumours: a review of parameters of the linear-quadratic model, derived from clinical radiotherapy studies. Rad Oncol 2018;13(1):1-11.

[25] Durante M, Paganetti H. Nuclear physics in particle therapy: a review. Rep Prog Phys 2016;79(9):096702.

[26] Pfuhl T, Horst F, Schuy C, Weber U. Dose build-up effects induced by delta electrons and target fragments in proton Bragg curves-measurements and simulations. Phys Med Biol 2018;63(17):175002.

[27] Mairani A, Dokic I, Magro G, Tessonnier T, Bauer J, Böhlen T, Ciocca M, Ferrari A, Sala P, Jäkel O, et al. A phenomenological relative biological effectiveness approach for proton therapy based on an improved description of the mixed radiation field. Phys Med Biol 2017;62(4):1378.

[28] Grün R, Friedrich T, Traneus E, Scholz M. Is the dose-averaged LET a reliable predictor for the relative biological effectiveness? Med Phys 2019;46(2):1064-74. 